\title{
Unified Link Layer Security Design for IP Encapsulation using Unidirectional Lightweight Encapsulation over Satellites
}

\author{
H. Cruickshank ${ }^{1}$, L. Liang1, P. Pillai ${ }^{2}$, M. Noisternig ${ }^{3}$, B. Collini-Nocker ${ }^{3}$, G. Fairhurst ${ }^{4}$ \\ ${ }^{1}$ CCSR, University of Surrey, Guildford, UK; ${ }^{2}$ MSCRC, University of Bradford, UK \\ ${ }^{3}$ University of Salzburg, Austria; ${ }^{4}$ ERG University of Aberdeen, UK
}

Keywords: Security, ULE, DVB, Satellites

\begin{abstract}
There is growing interest in providing multimedia and broadband access over satellites. However there are several technical challenges need to be addressed. One challenge is security in terms of understanding threats and providing an effective security system. Also this paper presents a ULE security solution using ULE mandatory extension headers. The design issues and choices are discussed. The detailed security header format is described together with processing sequence in both transmitter and receiver sides.
\end{abstract}

\section{INTRODUCTION}

There probably exists no other application of satellite technology that is as well known as satellite broadcasting [1], [2]. The Digital Video Broadcasting (DVB) system has been designed with a modular structure, based on independent subsystems, so that a variety of DVB systems (such as DVB-S: satellite, DVB-T: terrestrial, DVB-C: cable) could maintain a high level of interoperability. The challenge for the next generation of satellite access systems is to define a common basis for efficient integration of satellites in IP-centric telecommunication networks.

ULE has been defined by the IETF IP over DVB working group [3] for transmitting IP datagrams over MPEG-2 Transport Streams. It can be used for transporting of IPv4 packets, IPv6 packets, and even bridged MAC frames over a DVB-S/RCS satellite. This data is encapsulated in a ULE Sub-Network Data Unit (SNDU) which is then directly placed into the MPEG-2 Transport Stream before transmission over the satellite [4].

Figure 1 shows the packet format for an ULE SNDU. The Receiver Destination Network Point of Attachment (NPA) address field is optional. The Destination address absent field (D) indicates the presence of the destination address field. When the destination address is omitted, the DVB receiver and gateway may use the embedded layer 3 address (IP address in this case) for filtering packets addressed to them. The Type field indicates the type of the payload carried by the ULE SNDU, for example 0x0800 for IPv4.

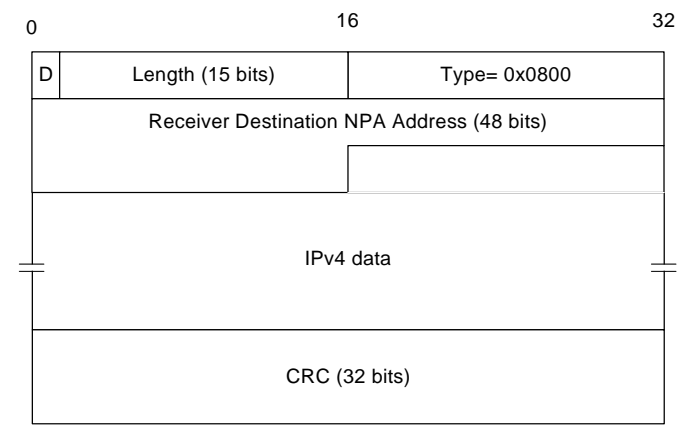

Figure 1. ULE SNDU format for an IPv4 datagram [4]

Each SNDU also carries a 32-bit Cyclic Redundancy Code (CRC) field to protect the SNDU from undetected reassembly errors and errors introduced in the processing and transit over the DVB network [4].

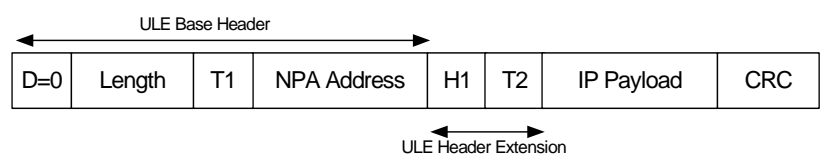

Figure 2. ULE SNDU packet with header extension [4]

An extension format for the ULE SNDU has also been defined so that new extension headers can be added to the ULE SNDU [4]. Figure 2 shows the packet format for an ULE SNDU with one extension header, H1. The type field, T1 in the ULE base header would specify the type of the extension header, while the type $\mathrm{T} 2$ in the extension header would specify the type of payload carried by the SNDU [4].

The rest of the paper is organized as follows. In Section 2, the ULE mechanism used for transmitting IP over DVB is explained. The ULE packet formats and header extension capabilities are also discussed. In Section 3, the threats and the security requirements for IP services over DVB are discussed. The various security and practical issues arising in the design of a security extension for the ULE are discussed in detail in Section 4. The new proposed unified ULE extension header to provide the various security requirements is explained in Section 5. Finally, conclusions are discussed in section 6. 


\section{Threats AND SECURITY REQUIREMENTS}

\section{$2.1 \quad$ Threats}

Passive threats are the simplest type of network threat possible on any network [5]. This includes eavesdropping and traffic analysis (i.e. monitoring of transmissions) with a goal to obtain information about the communicating parties and other information about the data that is being transmitted. In broadcast networks such as DVB that utilise widely available low-cost physical layer interfaces, such passive threats are a major concern. One example of such a passive threat is an intruder monitoring the MPEG-2 transmission broadcast and then extracting the data carried within the link. Another example is an intruder trying to determine the identity of the communicating parties and the volume of their traffic by sniffing the layer 2 (and possibly layer 3) addresses. While this is a well-known issue when dealing with network security, however, it is more of a problem in the case of broadcast networks such as MPEG-2 based DVB-S/RCS satellite networks because of the easy and wide availability of receiver hardware and the large geographical coverage of the satellite network.

Active threats (or attacks) on the other hand are in general more difficult to implement successfully than passive threats and usually require more sophisticated resources and may also require access to the transmitter. Within the context of MPEG-2 transmission networks, examples of active attacks are:

- Masquerading: An entity pretends to be a different entity. This includes masquerading other users and subnetwork control plane messages.

- Modification of messages in an unauthorised manner.

- Denial-of-Service (DoS) attacks: When an entity fails to perform its proper function or acts in a way that prevents other entities from performing their proper functions.

- Replay attacks: When an intruder sends some old (authentic) messages to the receiver. In the case of a broadcast link, access to previous broadcast data is easy.

The active threats mentioned above are major security concerns for the Internet community [6]. Masquerading and modification of IP packets are comparatively easy in an Internet environment, whereas such attacks are in fact much harder for DVB-S/RCS satellite broadcast links. Hence, while the use of sequence numbers is mandatory in IPsec [7], it may not be required all the time for the satellite link. This is further reflected in the security requirements for Case 2 and 3 in Section 3.2 below.

In a DVB-S/RCS satellite network, the PID associated with an Elementary Stream (ES) can be modified for example in some systems by reception of an updated SI table, or in other systems until the next announcement/discovery data is received. Hence an attacker that is able to modify the content of the received multiplex (e.g., replay data and/or control information) could inject data locally into the received stream with an arbitrary PID value.

\section{$2.2 \quad$ Threat Cases}

The security threats for DVB-S/RCS satellite can be abstracted into the following three cases:

- Case 1: Passive threat. Here the intruder monitors the ULE broadcasts to gain information about the ULE data and/or tracking the communicating parties' identities (by monitoring the destination NPA address). In this scenario, measures must be taken to protect the ULE payload data and the identity of ULE Receivers.

- Case 2: Locally conducting active attacks on the MPEGTS multiplex. Here an intruder is assumed to be sufficiently sophisticated to override the original transmission from the ULE encapsulation gateway and deliver a modified version of the MPEG-TS transmission to a single ULE Receiver or a small group of Receivers (e.g., in a single company site). The DVB-S/RCS satellite network operator might not be aware of such attacks. Measures must be taken to ensure ULE data integrity and authenticity, and preventing replay of old messages.

- Case 3: Globally conducting active attacks on the MPEG-TS multiplex. This assumes a sophisticated intruder able to override the whole MPEG-2 transmission multiplex. The requirements are similar to case 2. The MPEG-2 transmission network operator can usually identify such attacks and provide corrective action to restore the original transmission.

For both Cases 2 and 3, there can be two sub-cases:

- Outsider attacks, i.e., active attacks from adversaries without knowledge of the secret material.

- Insider attacks, i.e., active attacks from adversaries within the network with knowledge of the secret material.

In terms of priority, Case 1 is considered the major threat in DVB-S/RCS satellite systems. Case 2 is considered a lesser threat, appropriate to specific network configurations, especially when vulnerable to insider attacks. Case 3 is less likely to be found in an operational network, and is expected to be noticed by the DVB-S/RCS satellite operator. It will require restoration of the original transmission. The assumption being that physical access to the network components (multiplexers, etc.) and/or connecting physical media is secure. Therefore, Case 3 is not considered further in this paper.

\subsection{Security Requirements for IP over MPEG-2 TS}

From the threat analysis in the above section, the following security requirements can be derived:

- Req 1: Data confidentiality MUST be provided by a link that supports ULE Stream Security to prevent passive attacks and reduce the risk of active threats. 
- Req 2: Protection of Layer 2 (L2) NPA address is OPTIONAL. In broadcast networks, this protection can be used to prevent an intruder tracking the identity of ULE receivers and the volume of their traffic.

- Req 3: Integrity protection and source authentication of ULE Stream data are OPTIONAL.

- Req 4: Protection against replay attacks is OPTIONAL.

- Req 5: L2 ULE Source and Receiver authentication is OPTIONAL. This can be performed during the initial key exchange and authentication phase, before the ULE receiver can join a secure session with the ULE Encapsulator (i.e., the ULE source). This could be either unidirectional or bidirectional authentication based on the underlying key management protocol.

The following general requirements are also important for all threat cases when providing link-layer security for DVBS/RCS satellite system:

- GReq (a): ULE key management functions MUST be decoupled from ULE security services such as encryption and source authentication. This allows the independent development of both systems.

- GReq (b): Support SHOULD be provided for automated as well as manual insertion of keys and policy into the relevant databases.

- GReq (c): Algorithm agility MUST be supported. It should be possible to update the crypto algorithms and hashes when they become obsolete without affecting the overall security of the system.

- GReq (d): The security extension header MUST be compatible with other ULE extension headers. The method must allow other extension headers (either mandatory or optional) to be used in combination with a security extension. It is RECOMMENDED that these are placed after the security extension header. This permits full protection for all headers. It also avoids situations where the SNDU has to be discarded on processing the security extension header, while preceding headers have already been evaluated. One exception is the Timestamp extension that SHOULD precede the security extension header [8]. In this case, the timestamp will be unaffected by security services such as data confidentiality and can be decoded without the need for key material.

Examining the threat cases in Section 2.2, the security requirements for each case can be summarised as follows:

- Case 1: Data confidentiality (Req 1) MUST be provided to prevent monitoring of the ULE data (such as user information and IP addresses). Protection of NPA addresses (Req 2) MAY be provided to prevent tracking ULE Receivers and their communications.

- Case 2: In addition to Case 1 requirements, new measures MAY be implemented such as authentication schemes using Message Authentication Codes, digital signatures, or Timed Efficient Stream Loss-Tolerant
Authentication (TESLA) [9] in order to provide integrity protection and source authentication (Reqs 3 and 5). In addition, sequence numbers (Req 4) MAY be used to protect against replay attacks. In terms of outsider attacks, group authentication using Message Authentication Codes can provide the required level of security (Reqs 3 and 5). This will significantly reduce the ability of intruders to successfully inject their own data into the MPEG-TS stream. However, scenario 2 threats apply only in specific service cases, and therefore authentication and protection against replay attacks are OPTIONAL. Such measures incur additional transmission as well as processing overheads. Moreover, intrusion detection systems may also be needed by the MPEG-2 network operator. These should best be coupled with perimeter security policy to monitor common DoS attacks.

- Case 3: As stated in Section 2.3, the requirements here are similar to Case 2, but since the MPEG-2 transmission network operator can usually identify such attacks, the constraints on intrusion detections are less than in Case 2.

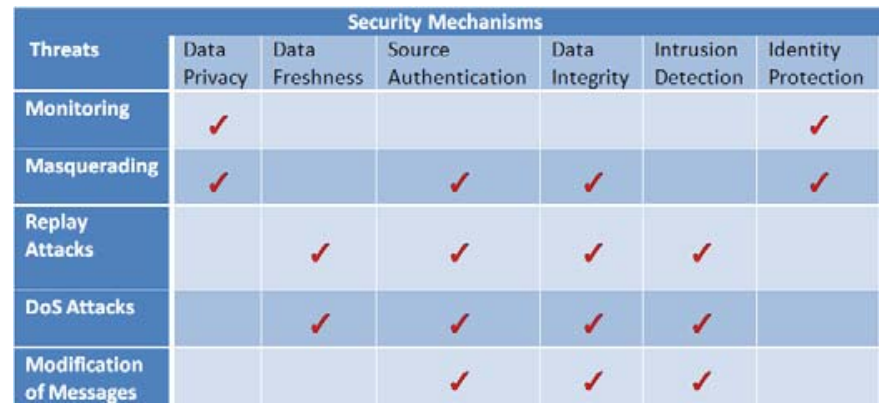

Table 1: Security techniques to mitigate network threats

Table 1 shows the threats that are applicable to ULE networks, and the relevant security mechanisms to mitigate those threats.

\section{SECURITY EXTENSION DESIGN IsSUES}

This section highlights a number of security and practical issues in the design of a security extension for the ULE protocol. Many of these issues stem from the requirement of identity protection, which is a novelty compared to other security protocols known. Other issues arise from network architectural properties under which the ULE protocol executes.

\subsection{Security Issues}

Applying standard cryptographic and engineering practice is insufficient to meet the security goals required for a ULE security extension. This is particularly due to the introduction of identity protection. Using traffic analysis techniques, a passive adversary may be able to link related packets to a single connection, potentially enabling him to deduce the real destination address, even when precluded from decoding the destination NPA address field. Various possible attacks and solutions are discussed in the following. 


\subsubsection{Address Hiding}

Hiding an SNDU's real destination NPA address from an adversary is arguably the most important step on providing identity protection. The use of temporary addresses was suggested in [10]. Subsequently, [11] determined a number of practical issues, such as the problem of allocating and distributing unique temporary addresses within the system. From a security point of view, temporary addresses do not provide adequate identity protection, as a passive adversary may easily link different SNDUs to the same connection.

By encrypting the destination NPA address within the SNDU, an attacker is effectively denied from gaining information by monitoring addresses. In [10] it was shown that the probability of falsely accepting an SNDU due to decryption with the wrong key is very low, and close to typical packet-error ratios on the ULE link, while legitimate receivers will always be able to correctly decode the protected destination NPA address.

\subsubsection{SID Sharing}

The security extension described in this document relies on Security Identifiers (SIDs) in the protocol to look up Security Associations (SAs) (see section 4.2 on practical issues). Since SIDs represent static connection-dependent parameters, they are similar in form to temporary addresses. The suggestion in [10] therefore was to omit the SID for identity-protected SNDUs, and instead use a kind of trial-and-error approach to figure out a matching key. This is problematic for several reasons: it increases the chance of erroneous acceptance of an SNDU; it introduces an element of indeterminism in the decoding process; and it assumes the existence of an encrypted address field containing pseudo-random data irrespective of whether identity protection is used in a security extension header or not.

Instead, a solution could be what we call "SID sharing". The idea is that if different receivers use the same SID value for their identity-protected communication, a suitable security extension header layout (see section 4.2.2) assures that they will not interfere with each other's communication, while identity protection is provided in the sense that an adversary can figure out an SNDU's real destination neither by monitoring the encrypted addresses nor by monitoring the SID values.

SID sharing could be enforced either by manual configuration, by central configuration via a group controller (such as in multicast and unidirectional settings), or by selecting SIDs from a pool prioritized for SID sharing.

\subsubsection{Sequence Numbers}

Sequence numbers, frequently used to attain the security goal of replay protection, and as nonces for the counter (CTR) encryption mode of operation, resemble unkeyed connection states that an adversary may track to link packets to different connections. It is one of the reasons why we do not mandate sequence numbers for our security extension protocol, in contrast to IPsec and numerous other security protocols.
To retain the goal of replay protection, one solution is encrypting sequence numbers using standard ECB mode (i.e. encrypt as one block of data). A receiver first decrypts the blinded value within the protocol to check for a replay, and then may use either the encrypted value as an initialization vector for the CBC mode of operation, or the decoded sequence number for the CTR mode (with the internal counter increased by one). The disadvantage is a slightly higher protocol overhead compared to un-encoded sequence numbers.

Another solution is to use connection-independent timestamp values. Depending on the resolution, timestamps may or may not provide perfect replay protection. The drawback is a higher protocol overhead, including the need for synchronized clocks.

\subsubsection{Stateful Cryptographic Algorithms}

Stateful cryptographic algorithms are problematic for manually keyed configurations when devices cannot retain their cryptographic states across device restarts (due to power or device failures, etc.). Reuse of sequence numbers for the CTR mode renders all encryption insecure. Receiver devices may be vulnerable to replay attacks if they do not remember prior lower bounds for sequence numbers. If devices cannot store their cryptographic state in non-volatile memory, it is advised that they resort to non-stateful schemes, such as the randomized CBC mode for encryption, or the use of timestamps for replay protection (if replay protection is desired).

\subsubsection{Nonces}

Many stateful schemes, such as the CTR mode of operation, require nonces as part of their input. As mentioned before, nonces must never be re-used under the same key. To provide this guarantee, particularly under group communication where the encryption key is shared among several group members, some source identifier must be incorporated into the construction of the nonce.

Within ULE networks, the PID may be used as a source identifier, but this is not reliable. A device may receive data from different MPEG-2 multiplexes, which both may allocate PID values independently. Furthermore, multiplexors within the network may transparently re-number PID values. This is a problem for receivers as they require the originating PID value for reconstructing nonces.

One solution to circumvent these issues is to assign a unique sender identifier to each legitimate transmitter under the same key [8]. To avoid the problem of associating an MPEG-2 TS with a sender identifier, the latter is included explicitly as part of the nonce in each SNDU. This means that the nonce field (e.g., sequence number) may get enlarged by the size necessary to support a predefined maximum number of different senders sharing a key. The other caveat of this approach is the problem of generating and distributing unique sender identifiers.

\subsubsection{Scope of Authentication}

Reliable protection against modification of data and masquerading attacks requires both sender and receiver 
identifiers to be authenticated in addition to the payload. This is an issue for the ULE protocol because it does not exhibit a source identifier field, and the PID of an underlying MPEG-2 TS cell does not depict a unique and reliable identifier. Without authenticating the source, an active attacker may rewrite the PID to appear from a different transmitter under the same encryption key.

Above problems arise only in configurations with multiple senders sharing a key, and when PID re-numbering cannot be ruled out. Therefore, it seems reasonable to let PID authentication be optional, and the PID may be part of a pseudo-header for authentication in such case.

\subsection{Practical Issues}

\subsubsection{Security Association (SA) Lookup and Re-Keying}

Typically, upon creation of a secure connection, connection dependent parameters such as algorithms and key lengths are maintained in a state called Security Association (SA) at each side. Volatile parameters such as sequence numbers or initialization vectors are explicitly provided in the security protocol. Frequently, L2 security protocols do not provide means to multiplex several secure connections over a link. For example, MPE [4] just contains a bitfield indicating whether encryption is used or not. Supporting only a single SA is justified when a receiver device can directly communicate with a master (or base station) solely. However, in a meshed network devices may form virtual LANs by sharing a SA, and a single device may be part of more than one virtual LAN. A Security Identifier (SID) may assist a receiver device in looking up the correct SA. This approach, taken for example within the IPsec protocols in the form of the Security Parameter Index (SPI) [7], also allows for easy update of keys (re-keying) by simply switching to a new SA with a different SID. On the other hand, protocols not supporting a SID must employ one or more key bits to indicate a turn-over to new keys.

The solution presented in this document follows the IPsec approach of including a SID in the security extension for SA lookup. However, a straightforward use may preclude future communication in certain connection scenarios. ULE security aims to support connections for which a return channel is not available (unidirectional links), or for which use of the return link does not scale (multicast). Note that IPsec only supports manually keyed multicast settings. The problem is that a receiver that failed to receive key update messages will not be able to identify future packets with a new SID, and therefore ultimately lose connectivity. This is not a problem when key bits are used. An earlier suggestion therefore was to use such bits in addition to the SID [10]. This raises other practical concerns, such as how many key bits to use, and where to put these bits in the protocol without introducing reserved bits for alignment. Fortunately, the advantages of both approaches can be combined very elegantly: instead of adding key bits, a SA (via the key management protocol) can define a set of two or more SIDs through which are cycled whenever a key update occurs. In fact, because the number of SIDs need not be pre-defined, the solution is much more flexible than using key bits.
For the ULE security extension header, a SID size of 16 bits has been selected. This seems like a good compromise between conserving valuable protocol space and providing sufficient flexibility. Note that the IPsec protocols' SPI field is much larger with a size of 32 bits; this is justified with the fact that these are end-to-end security protocols, potentially supporting many different L3 connections, while on L2 users are expected to rarely support more than one or a few different secure connections.

\subsubsection{Position of the Encrypted Address}

Encryption of the destination NPA address for identity protection can be derived directly in the ULE base header. This has a couple of disadvantages: hardware address filtering for receiver devices supporting identity protection is precluded; "legacy" receiver devices (those not supporting the security extension) might not be able to filter identityprotected SNDUs with probability $1 / 2$ due to the multicast/broadcast bit set within the destination NPA address; and receiver devices must buffer the encrypted address until the security extension header is evaluated.

The ULE protocol allows omitting the destination NPA address in its base header by setting the $\mathrm{D}$ bit to one. This turns out to be a useful feature for identity protection, as it enables moving the encrypted address into the ULE security extension header. Besides allowing for a more coherent design, and not changing the semantics of the address field, the only drawback is that receiver devices accepting SNDUs with the D bit set to one will have to evaluate all identityprotected SNDUs up to the security extension header. When receiver processing overhead is an issue, one solution for legacy devices is to employ a policy enforcing SNDUs to be sent and received with a destination NPA address present. Another solution is to refer to connections using identity protection as a single multicast group, using the multicast group's address within the ULE base header in addition to a receiver's encrypted address in the security extension header. This is particularly useful for GSE where the base header's label type field allows for a 3-byte label format in place of the 6-byte address.

\subsubsection{SID Selection}

When identity protection is used for a connection, receivers assume the encrypted destination address at a certain position within the security extension header, based on the SID value found. If the data at that position is not sufficiently random, encrypted address decoding errors may not be uniformly distributed, and guarantees about low false acceptance rates of SNDUs may hence become void. A first step to remedy this problem is to keep the encrypted destination NPA address at a fixed position in the security extension header, in front of the SA-dependent data. Furthermore, SID values for identity protection should be strictly separated from those used for other secure connections within a network, i.e., there should be two pools of SID values, one for SAs with identity protection enabled, and one for those without.

\subsubsection{Position of the MAC}

The Message Authentication Code (MAC), while logically part of the security extension header, is realized as a trailer to 
the extension header, directly following the PDU. This has the advantage of "online" computation, which means that an encapsulator can derive the MAC during the process of transmitting an SNDU, and as soon as it has finished sending the PDU it can simply attach the MAC. This is in similar spirit to the CRC, which can also be regarded as a trailer to the ULE base header.

\section{SECURITY EXTENSION HEADER FOR ULE}

\subsection{Security Extension Header Format}

The proposed security extension shown in Figure 3 aims to secure the transmission of user traffic over MPEG-2 Transport Streams. This security extension is a standard extension header as described in Section 5 of RFC 4326 [4] and does not affect the ULE base protocol. This security extension header is a mandatory ULE extension header. This means that a receiver MUST process this header before it processes the next extension header or the encapsulated PDU, otherwise the entire SNDU should be discarded.

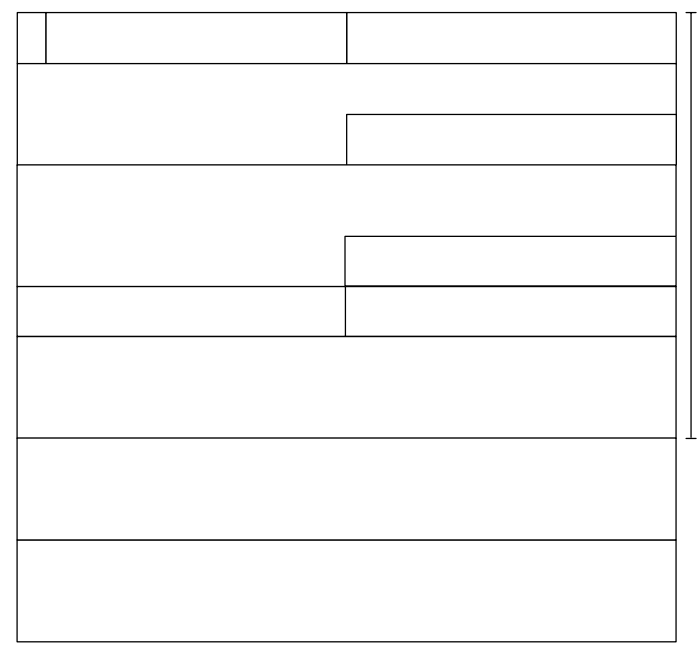

Figure 3: General SNDU format with Security extension header

In Figure 3, the Type field in the base header denotes that a mandatory security extension header is present. The receiver destination NPA address is optional. After the base ULE header the security extension header follows. This header contains the ULE-Security-ID, the encrypted destination address and the encrypted Next-Type field that denotes the type of the enclosed PDU. The higher-layer PDU is encrypted and then encapsulated in the SNDU. This is followed by the optional SA-Dependant-data field (for example, sequence Onumber) and optional Message Authentication Code (MAC) field. The format of the Destination Address Absent field (D), eragitength5fbitsthe Type field and the Receiver Destination NPA address field are defined by ULE [4].

4.1.1

Destination Address absent (

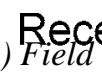

The most significant bit of the Length Field carries the value of the Destination Address Absent Field (D) as defined by ULE [4]. When D is set to 0 , it indicates the presence of the
Destination Address Field while D set to 1 indicates that a Destination Address Field is not present.

\subsubsection{Length Field}

A 15-bit Length field denotes the length, in bytes, of the SNDU counted from the byte following the Type field, up to and including the CRC [4].

\subsubsection{Type Field}

The 16-bit Type field of the ULE base header indicates that a security extension header follows subsequently.

\subsubsection{Destination NPA Address Field}

The SNDU Destination Address Field is optional. This field MUST be carried when field $\mathrm{D}$ is set to 0 and may be omitted when $\mathrm{D}=1$ [4].

\subsubsection{ULE-Security-ID Field}

A 32-bit security identifier, the ULE-Security-ID similar to the SPI used in IPsec has been added to uniquely identify the secure session. This ULE-Security-ID represents the security association between the MPEG-2 transmitter and receiver for a particular session and indicates the keys and algorithms used for encrypting the data payload and calculating the MAC. The ULE-Security-ID is used by a receiver to filter PDUs in combination with the encrypted destination address.

\subsubsection{Encrypted Destination Address field}

This field is only present if the identity protection service is used (determined by the SPs selected). In that case, SNDUs do not contain a 48-bit NPA destination address in the ULE base header (i.e., they have the $\mathrm{D}$ bit set to 1 ), but the address will appear in the security extension header's Encrypted Destination Address field instead, where it will be encrypted subsequently (along with the payload data).

\subsubsection{SA-Dependant-Data Field}

This optional SA-Dependant-data field may contain any information that is specific to the particular SA. An optional sequence number may be present in this field to prevent replay attacks. The gateway monotonically increments this sequence number when it sends a packet to the receiver and the receiver verifies the correct sequence number and must discard all SNDUs which do not match. If an adversary tries to inject or replay old packets the sequence number would not match. This would result in discarding the packet. SNDU reordering is not permitted on ULE links, and therefore any accidental reordering of segments will result in discard. The length of the Sequence Number field is defined by the SA.

Initialisation vectors for decryption purposes may also be present in the SA-Dependant-Data field.

\section{1.尹ु6 Encrypted Type Field}

This mandatoryThpoefigedenestestetype of PDU that is encrypted and encapsulated in the Secure ULE SNDU. If another ULE extension header follows, then this type field

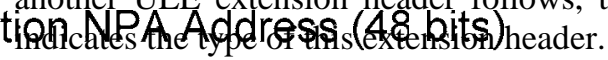

\subsubsection{Encrypted SNDU Payload}

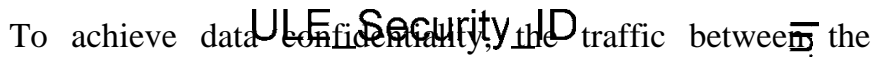
MPEG-2 TS transmitter (i.e. the ULE Encapsulator receiver needs to be encrypted. The network layer PD are 
first encrypted and then encapsulated in the secure ULE SNDU. The security associations between the two communicating points will describe the algorithms and keys used for encryption purposes.

Secure ULE does not impose the use of any specific encryption algorithm and should be able to support the commonly used algorithms including AES, 3DES etc.

\subsubsection{Message Authentication Code (MAC) Field}

To provide both data origin authentication and data integrity, a Message Authentication Code (MAC) is included in the extension header.

The MAC is calculated over the ULE security extension header and the encrypted data payload. The receiver calculates the MAC for the each received packet and compares it with the transmitted value. The two would not match in only 2 cases, firstly either there was an error during processing or transmission over the MPEG-2 Network, or secondly the packet has not been sent from an authenticated entity. In either case, the packet MUST be discarded. Hence the same MAC can be used for data origin authentication and to provide data integrity for transmission/processing errors.

\subsection{Transmitter Processing}

The following procedure is followed at the encapsulator for processing the security extension header for ULE:

- Upon reception of the higher layer PDU, the SPD is first queried to check the policy to be applied to the PDU. If security is needed then an SA must exist in the SAD (this is set by the key management system). The security parameters (like the encryption keys, the encryption algorithm, etc.) are retrieved from the SAD.

- Now the construction of the ULE SNDU begins. First, the header of the base protocol (and other extension headers if present) is added to the SNDU. If the SA requests identity protection, then the destination NPA address MUST be omitted from the base header

- The ULE-Security-ID for the security association between the transmitter and the receiver is added next.

- The destination address is now encrypted and added to the SNDU

- The SAD is consulted to determine the information that has to be added to the SA-Dependant-Data field. If the sequence number has to be added, then the corresponding sequence number is added to the SNDU.

- The type field and the higher layer PDU are first encrypted using the encryption key and the algorithm as indicated in the SAD and then added to the SNDU.

- The SAD is then checked to determine if the data origin authentication and data integrity has to be provided. If required, then the MAC has to be calculated. The MAC is calculated over the encrypted PDU (and other possible extension headers), the Security extension header and the secret key. The MAC is then added to the extension header in the SNDU.

- Finally, the CRC is calculated as defined in RFC4326 [4] and added to complete the SNDU

\subsection{Receiver Processing}

The following procedure is followed at the receiver for processing the security extension header for ULE:

- Upon reception of a Secure ULE SNDU, the Receiver first filters the received packets according to the receiver destination NPA address (if present).

- $\quad$ The CRC is verified as defined in RFC4326 [4].

- After this the base header and any extension headers up to the security extension header are evaluated.

- The Receiver then uses the ULE-SID to obtain the security policy from the SPD and subsequently the security associations between the transmitter and receiver and retrieve the data from the SAD. The SA is used to find the decryption keys and algorithms.

- Based on the SA, the receiver determines if the MAC is present or not. If present the next step would be to check the MAC to verify the authenticity and integrity of the received PDU. If the calculated MAC does not match the transmitted MAC, then the PDU is discarded.

- Based on the SA, the receiver determines if the sequence number is present or not. If present, it would then use the sequence number for filtering any out of-sequence packets.

- The encrypted destination address is decrypted and any packets not destined for the receiver are discarded.

- Finally the encapsulated payload will be decrypted and passed to the higher layers for processing.

\section{ConClusion}

For effective IP services over DVB satellite networks, a security solution must be addressed. This paper has analysed a set of threats and security requirements Satellite DVB networks and ULE encapsulation of IP traffic.

Also this paper has presented a ULE security solution using ULE mandatory extension headers. The design issues and choices are presented. The detailed security header format is described together with processing sequence in both transmitter and receiver sides.

\section{Acknowledgements}

This paper has been carried out within the framework of the SatNEx II (IST No. IST-027393) FP6 network of excellence.

\section{References}

[1] DVB Project home page: http://www.dvb.org 
[2] U. Reimers, et al "Special Issue: Satellite Broadcasting", International Journal of Satellite Communications, Vol. 18, No. 6, Nov.-Dec. 2000.

[3] IETF IP over DVB WG, http://www.ietf.org/html. charters/ipdvb-charter.html.

[4] G. Fairhurst, B. Collini-Nocker. "Unidirectional Lightweight Encapsulation (ULE) for transmission of IP datagrams over MPEG-2 Transport Stream (TS)”, IETF, RFC 4326, (2005).

[5] H. Cruickshank, P. Pillai, M. Noisternig, S. Iyengar. "Security requirements for the Unidirectional Lightweight Encapsulation (ULE) Protocol”, IETF, RFC 5458, (2009).

[6] S. Bellovin. "Security Problems in the TCP/IP Protocol Suite", Computer Communications Review 2:19, pp. 3248, (1989). http://www.cs.columbia.edu/ smb/

[7] S. Kent, K. Seo. "Security Architecture for the Internet Protocol”, IETF, RFC 4301, (2005).

[8] G. Fairhurst, B. Collini-Nocker. "Extension Formats for Unidirectional Lightweight Encapsulation (ULE) and the Generic Stream Encapsulation (GSE)", IETF, RFC 5163, (2008).

[9] A. Perrig, D. Song, R. Canetti, J. Tygar, B. Briscoe. "Timed Efficient Stream Loss-Tolerant Authentication (TESLA): Multicast Source Authentication Transform Introduction", IETF, RFC 4082, (2005).

[10] H. Cruickshank, P. Pillai, S. Iyengar. "Security Extension for Unidirectional Lightweight Encapsulation Protocol”, IETF, draft-cruickshank-ipdvb-sec-05 (expired), (2008).

[11] M. Noisternig, B. Collini-Nocker. "A lightweight security extension for the Unidirectional Lightweight Encapsulation (ULE) protocol”, IETF, draft-noisternigipdvb-ulesec-01 (expired), (2008). 